\title{
LOW REACTIVE LEVEL LASER THERAPY (LLLT) FOR THE TREATMENT OF HYPERTROPHIC SCARS AND KELOIDS. A RE-INTRODUCTION
}

\author{
*Shunji Fujii M.D. ${ }^{1}$, Toshio Ohshiro M.D., Ph.D. ${ }^{2}$, \\ Takafumi Ohshiro M.D. ${ }^{1}$, Katsumi Sasaki M.D. ${ }^{1}$, and Yuki Taniguchi M.D. ${ }^{1}$ \\ 1 Obshiro Clinic, Tokyo, Japan \\ 2 Japan Medical Laser Laboratory, Tokyo, Japan
}

\begin{abstract}
Low reactive Level Therapy ( LLLT ) is now well into its 30th decade, and its beneficial effects such as pain attenuation, accelerated or enhanced wound healing have been very well reported and investigated. For clinics such as ours which utilizes LLLT for medical treatments, LLLT for hypertrophic scars and keloids very quickly became, and remains, routine. We believed that many facilities and institutions have also been using LLLT for the treatment of hypertrophic scars and keloids, but to our surprise there were very few medical facilities using LLLT and practically none for the treatment of scars. Recently, starting in the 1990's, there have been several reports where High reactive Laser Treatment (HLLT) has been used in an attempt to treat hypertrophic scars and keloids. However no literature concerning LLLT for these lesions appears in MedLine, even though the treatment is safe and effective with no side effects and complications when compared to HLLT. The authors herein re-introduce the technique and protocol of LLLT which they have used for the past twenty years for the treatment of hypertrophic scars and keloids. We present a series of representative cases and discuss the most efficient treatment modality in combination with conventional treatment methods.
\end{abstract}

Key Words: LLLT, keloids, hypertrophic scars, GaAIAs diode laser, proximal priority treatment ( PPT )

\section{Introduction}

The treatment of hypertrophic scars and keloids has attracted controversy for decades. Though surgical intervention may be a simple option in many cases, surgery is absolutely contraindicated for keloids, and scar location and size may contraindicate surgery leaving no feasible options for the attending surgeon. Recently, starting in the 1990's, several reports have appeared where High reactive Laser Treatment (HLLT ) was used in an attempt to treat hypertrophic scars and keloids. However no literature concerning LLLT for these lesions appears in MedLine.

Low reactive level laser therapy (LLLT) for the treatment of pain attenuation and enhanced wound

Addressee for Correspondence:

Shunji Fujii M.D.

JR Shinanomachi-building 2F, 34 Shinanomachi, Shinjuku-

ku, Tokyo, Japan

$160-0016$

TEL: +81-3-3352-0046

FAX: +81-3-3354-1103

E-mail: info@jmll.co.jp healing was first presented well over 25 years ago. Many who utilize LLLT accept the efficacy of LLLT for those conditions and further experience with LLLT has led us to the treatment of hypertrophic scars and keloids. The authors have consistently believed that, due to the simplicity and efficacy of the treatment, many facilities and institutions have already been using this treatment modality. Literature-wise this does not seem to be the case. We and other LLLT users may have taken it for granted that everyone knew of this treatment modality and may have neglected to report their experience about it, or it may be that LLLT for the treatment of hypertrophic scars and keloids is not as well known or accepted by the general medical population as we thought it had been.

The authors would like to re-introduce the safe and effective treatment for hypertrophic scars and keloids using LLLT and present representative cases. The authors discuss the mechanism of LLLT for hypertrophic scars and keloids, and treatment regimen

Manuscript received: February 2008

Accepted for publication: March 2008 


\section{ORIGINAL ARTICLES}

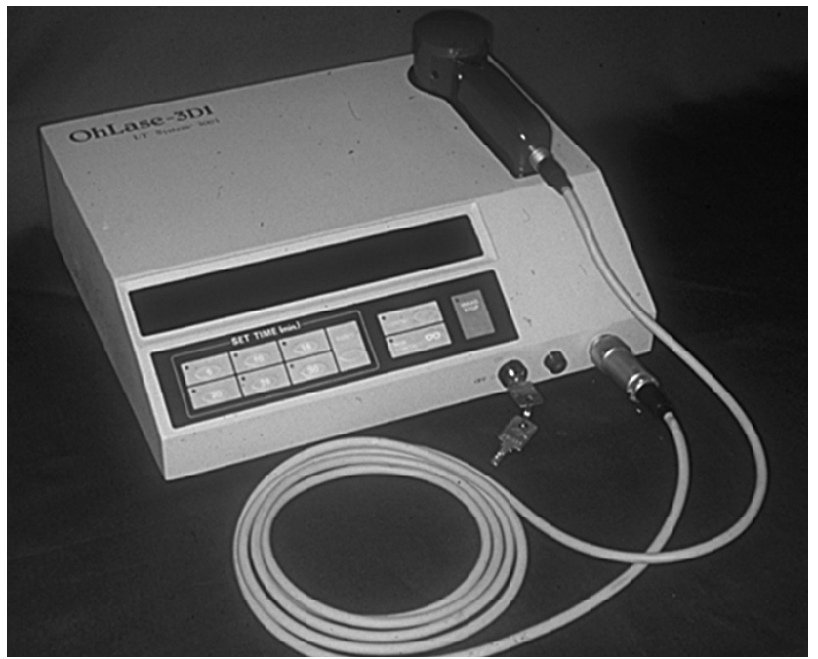

Fig. 1: 830nm GaAlAs diode laser, $60 \mathrm{~mW}$ continuous wave

which we believe is optimal in combination with other conventional and/or new treatment methods.

\section{Patients and Methods}

All patients presenting to Ohshiro Clinic are examined for their hypertrophic scars and keloids. Patients with hypertrophic scars indicative for surgical scar revision were advised to receive surgery. Patients with keloids or those with scars contraindicating surgery due to size, location and/or number of scars, and those patients refusing surgery were treated with LLLT. Patients were advised to receive treatment at least once every other week and if possible once a week. Each treatment session consists of direct local application of a $830 \mathrm{~nm}$, GaAlAs diode laser, the Oh-Lase 3D1 (Japan Medical Laser Laboratory, Tokyo, Japan) (fig. 1) to the lesion. Lesions are irradiated with the laser in the contact mode while the lesions are pinched firmly by the attending surgeon. Lesions are irradiated for $30 \mathrm{sec}-$ onds to 2 minutes per lesion depending on the size and number of lesions existing on the patient. No posttherapy restrictions are set for patients and they are free to go about with their normal daily activities. A local injection of triamcynolone acetate, the use of topical triamcynolone or the use of silicone adhesive dressing can be prescribed for the patient at the surgeon's discretion.

Hypertrophic scars and keloids are evaluated both subjectively and objectively for symptoms such as pain and itch, size, elevation of lesion, hardness and pliability, redness of lesion and the presence of contractures. Digital photographs are taken prior to the initial treatment and periodically after treatment has commenced.

\section{Cases}

\section{Case 1. 64 year old female (fig. 2a,b)}

Chief complaint: itchy and painful keloid of the lower abdomen

Onset and course: This patient received a total anterior hysterectomy and oophorectomy one year prior to presentation to our clinic. The keloid started to proliferate at 3 months post-operatively from the lower edge of her abdominal scar, growing slowly inferiorly. Pain on touch and spontaneous pruritis were present from the beginning. The patient sought medical treatment from her gynecologist where she was prescribed topical steroids and oral tranilast. The patient continued this treatment without success for 6 months and was extremely worried that the keloid might invade in to her genital area. She then came to our clinic.

Initial findings: The keloid was round, pear shaped with vertical diameter of $5 \mathrm{~cm}$ and horizontal diameter of $4 \mathrm{~cm}$ and slightly elevated. The texture of the keloid was brittle and hard. The lesion could not be folded and pinched as in normal skin. Contracture was seen at the upper margin of the keloid and hair growth from the keloid dictated that originally the area was intact, normal skin and not part of the abdominal scar.

Treatment: The patient was treated with LLLT for 2 minutes a session on a weekly basis. Due to the previous topical steroid therapy the epithelium of the keloid was atrophic and weak therefore no topical steroid was prescribed. Treatment by pinching was extremely painful at the start. By the fifth treatment spontaneous itching has subsided and after the 28th session the keloid has regressed and had changed its shape to a forked shape where three prongs extend from the body of the keloid inferiorly and slightly laterally. We believe that the tip of the prongs were the center of keloidal activity and the laser was applied mainly to the these tips and at the superior neck of the keloid. At the time of writing, the keloid has softened and can be folded between the prongs. Treatment is still painful but not as excruciating as in the initial treatments. Following the cessation of the initial pruritis and pain, the patient's treatment was reduced to a treatment on a bi-weekly basis at which rate treatment still continues. 


\section{PROFILE OF AUTHORS AND CO-AUTHORS}

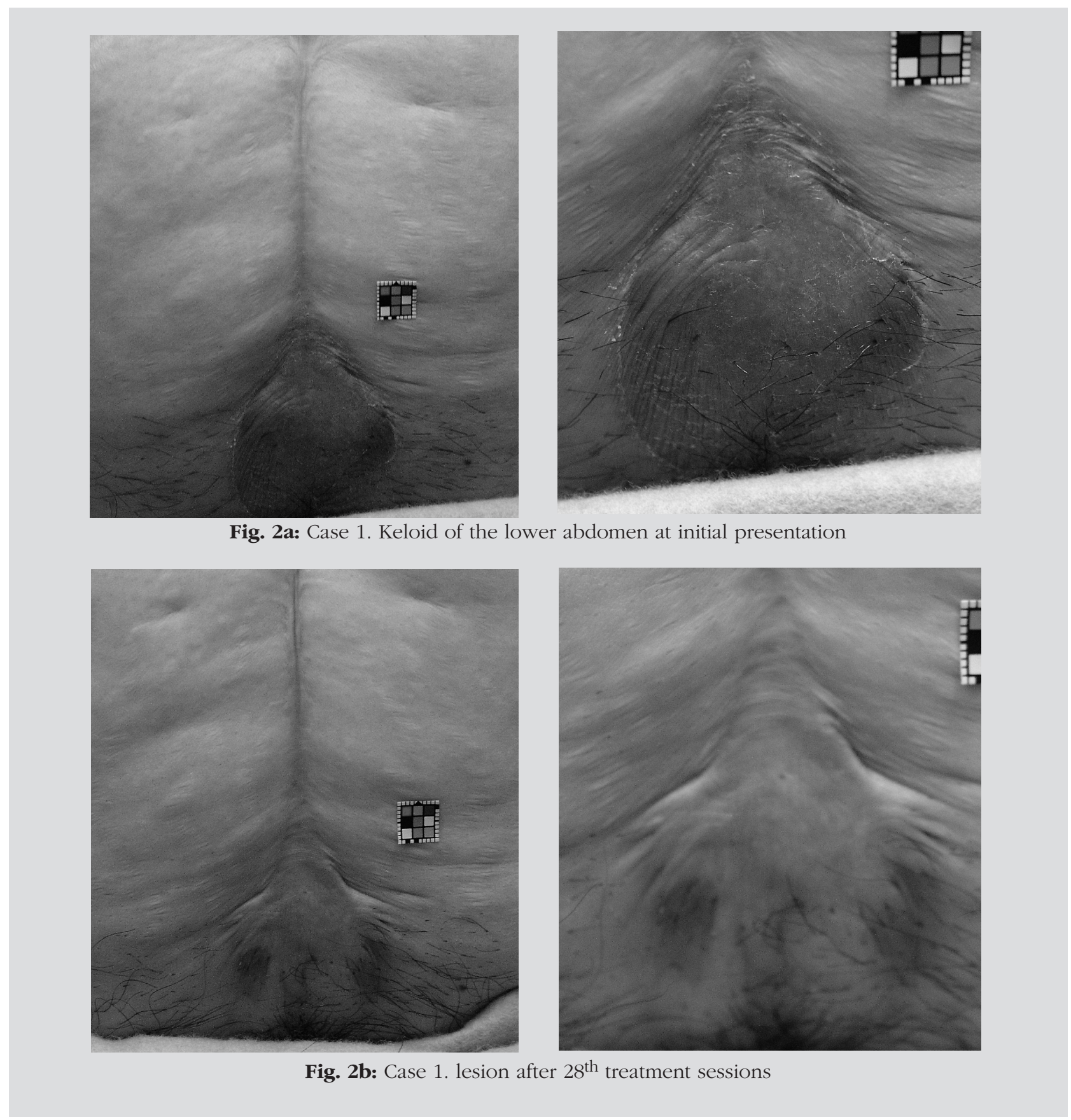

\section{Case 2. 37 year old female (fig. 3a,b)}

complaint: Keloid of the left scapular region

Onset and course: This patient had surgical removal of a benign skin lesion two years prior to presentation. At 6 months post-surgery she noticed elevation and itch of the surgical scar. On consultation at another dermatologist, the lesion was diagnosed as a hypertrophic scar and surgical revision was performed. Three months following scar revision the patient noticed the same itch returning and this time the lesion grew larger than the original scar and elevation reached roughly 1 $\mathrm{cm}$ within a period of one year. She was prescribed oral tranilast but no visible change of the lesion was seen.

Initial findings: The lesion was $3 \mathrm{~cm}$ in length and $2 \mathrm{~cm}$ wide. Suture marks of the scar revision could be seen in the middle of the lesion where it was the most rigid. However the lesion was elastic and could be bent.

Treatment: This patient was included in a study 


\section{ORIGINAL ARTICLES}
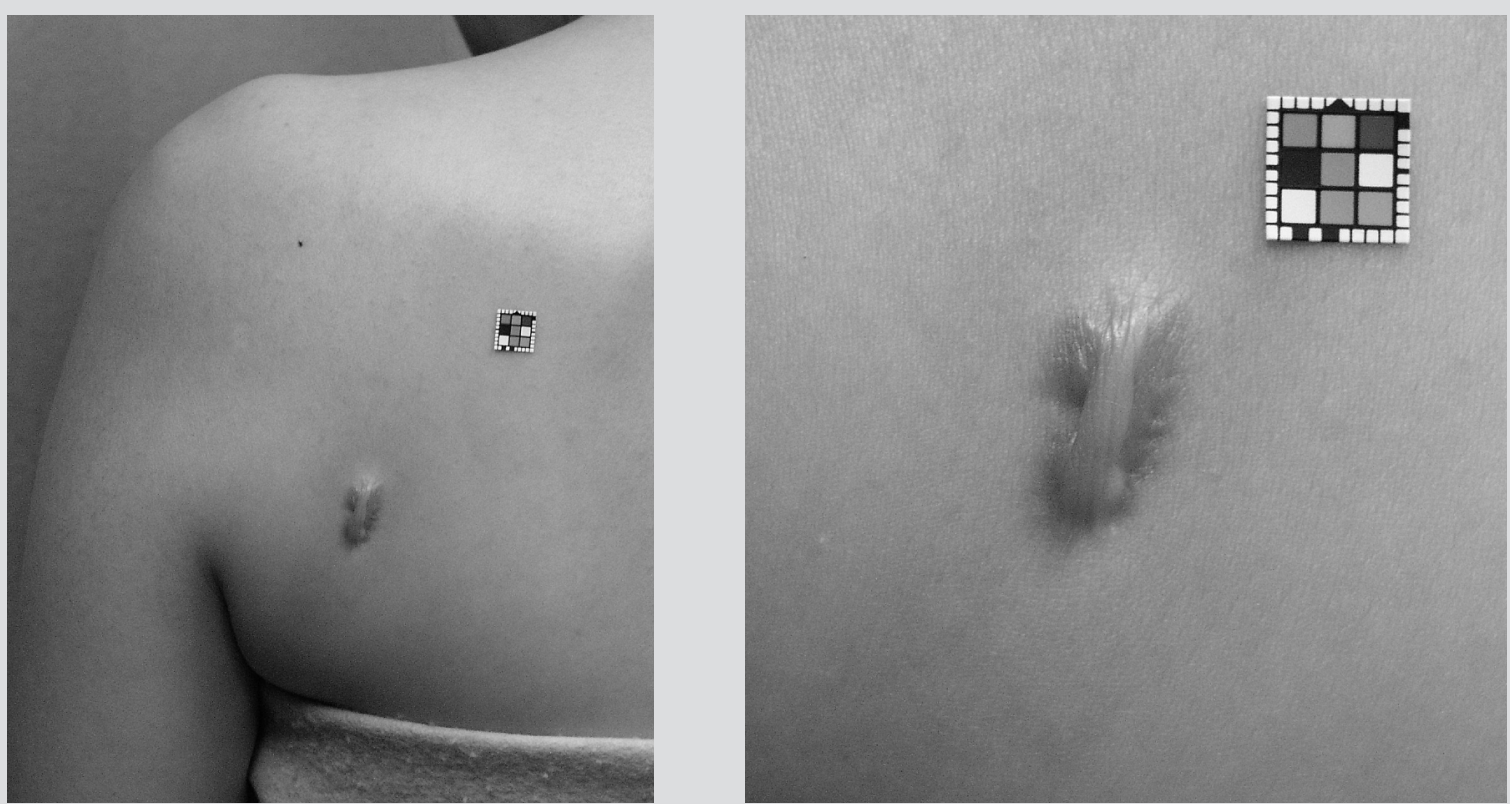

Fig. 3a: Case 2. Keloid of the left scapular region, at initial presentation
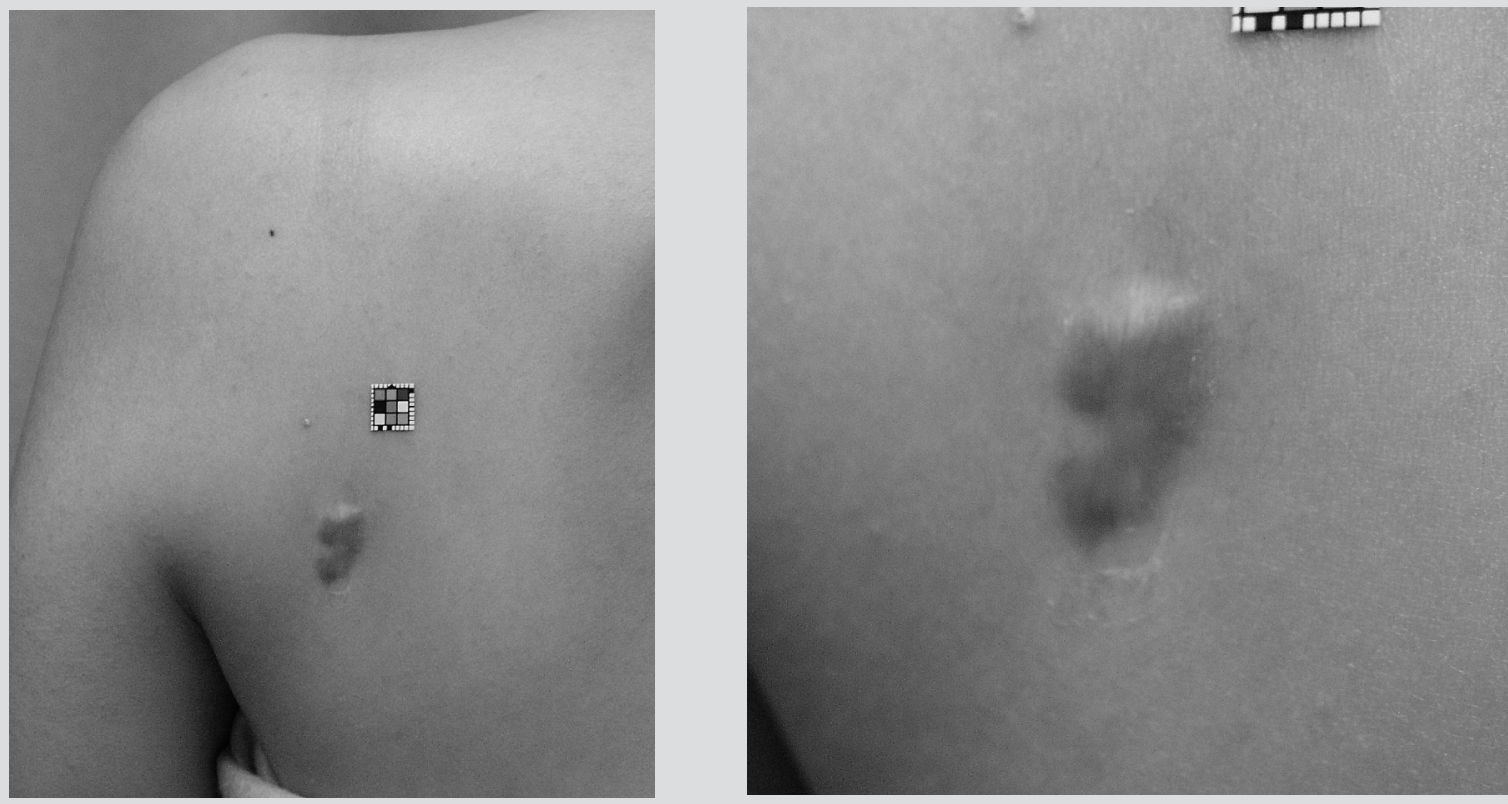

Fig. 3b: Case 2. lesion after $8^{\text {th }}$ treatment sessions

to find whether proximal laser irradiation to the neck as in the proximal priority treatment for pain would show favorable results compared to conventional local laser irradiation to the lesion. She was treated once a week or every other week depending on her work schedule. Topical steroids were prescribed. After the third treatment session, the patient reported complete cessation of any itching. By the eighth treatment the lesion had gradually softened and changed its shape and flattened to an elevation of $0.5 \mathrm{~cm}$. The patient is continuing this treatment regimen.

\section{Case 3. 30 year old female (fig.4 a,b,c)}

Chief complaint: Hypertrophic scarring of the face.

Onset and course: This patient was injured in a motorcycle accident while traveling in south east Asia. She suffered a deep laceration to the right portion of the upper lip and skin defect of the upper left cheek. After 3 months the scar of the upper lip had hypertrophied 


$\underline{L \Perp B} \quad$ ORIGINAL ARTICLES
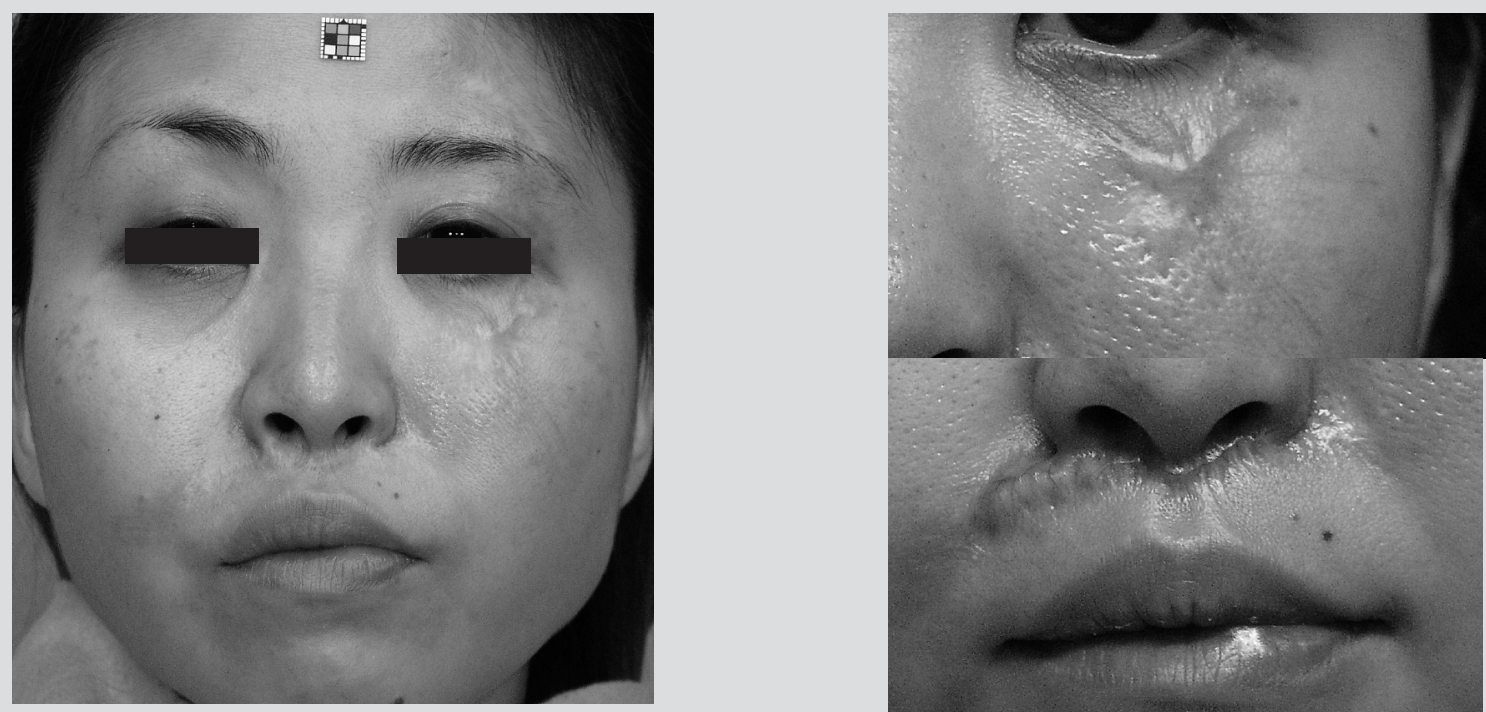

Fig. 4a: Case 3. hypertrophic scars, at initial presentation
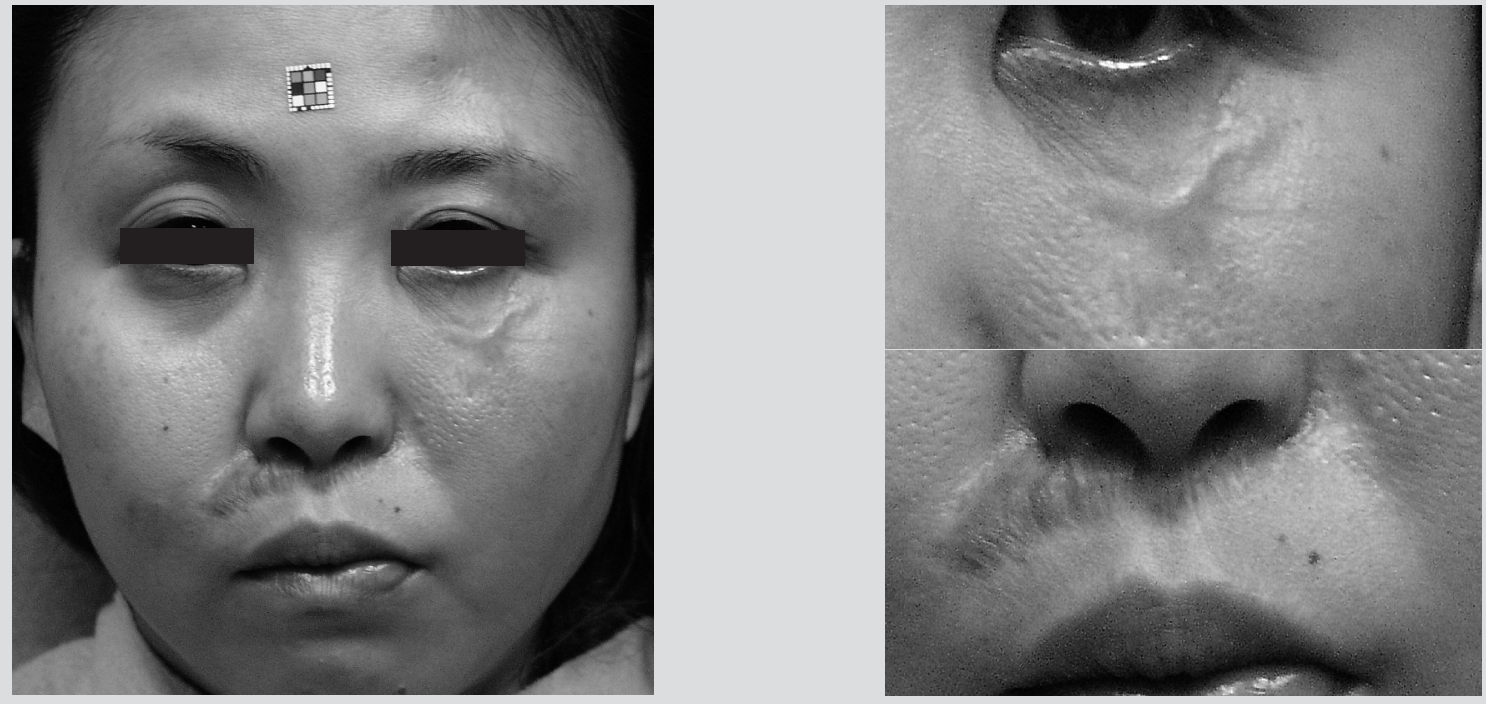

Fig. 4b: Case 3. after $23^{\text {rd }}$ treatment session, before revision of upper lip scar
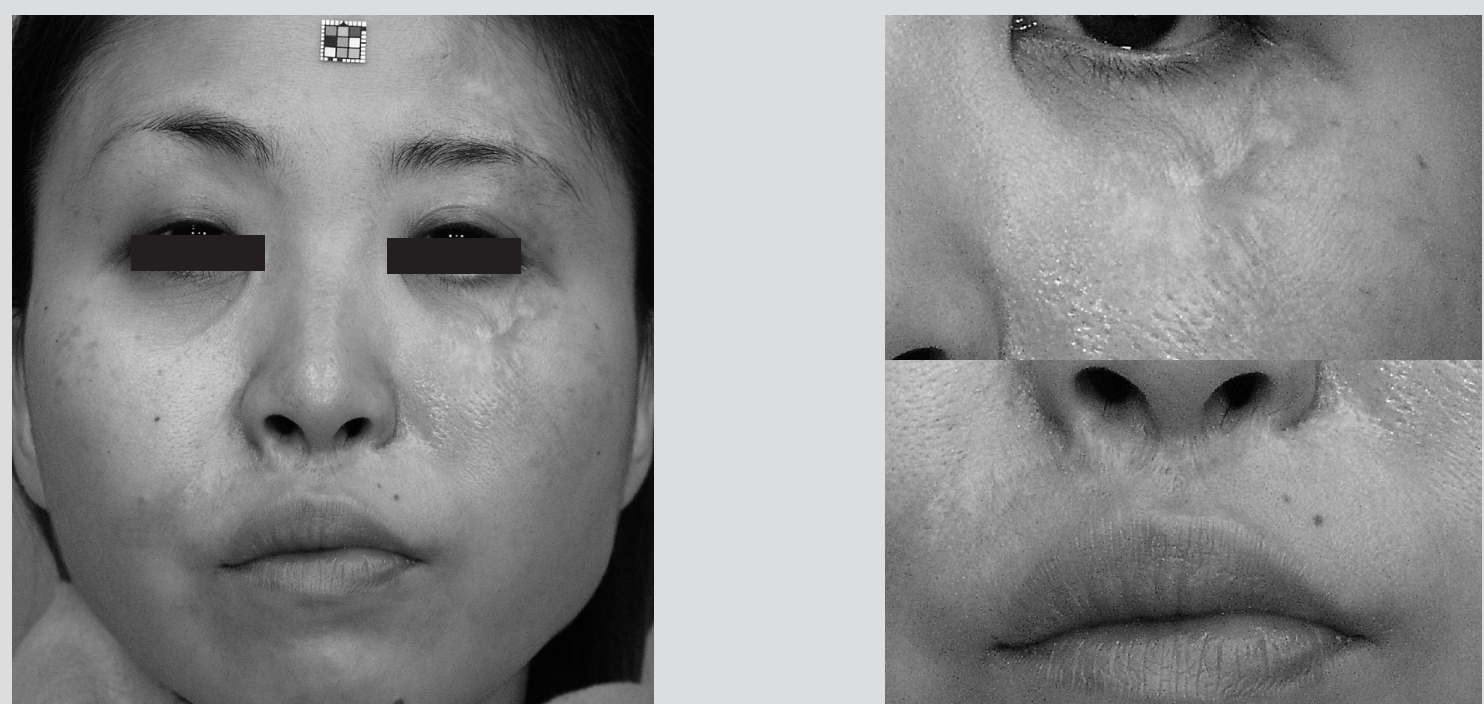

Fig. 4c: Case 3. after scar revision of upper lip and after $39^{\text {th }}$ treatment session 


\section{ORIGINAL ARTICLES}

and the skin defect of the cheek showed contracture. This patient originally presented at another plastic surgeons where she was referred to our clinic since the lesions were deemed inoperable.

Initial findings: The hypertrophic scar of the upper lip was $3.5 \mathrm{~cm}$ in length and $0.5 \mathrm{~cm}$ wide. Elevation was $0.5 \mathrm{~cm}$ at its highest point. The scar was hard and not pliable in any direction. Neither spontaneous pain nor itching was noted. The lesion on the left upper cheek showed only marginal elevation but the contracture caused slight ectropion of the left lower eyelid.

Treatment: The patient was treated two minutes a session, once a week for the first twenty sessions with only occasional absence from treatment. Topical steroids were used throughout the treatment. Although there were no gross dimensional changes of the lesion of the upper lip, dramatic softening of the lesion was
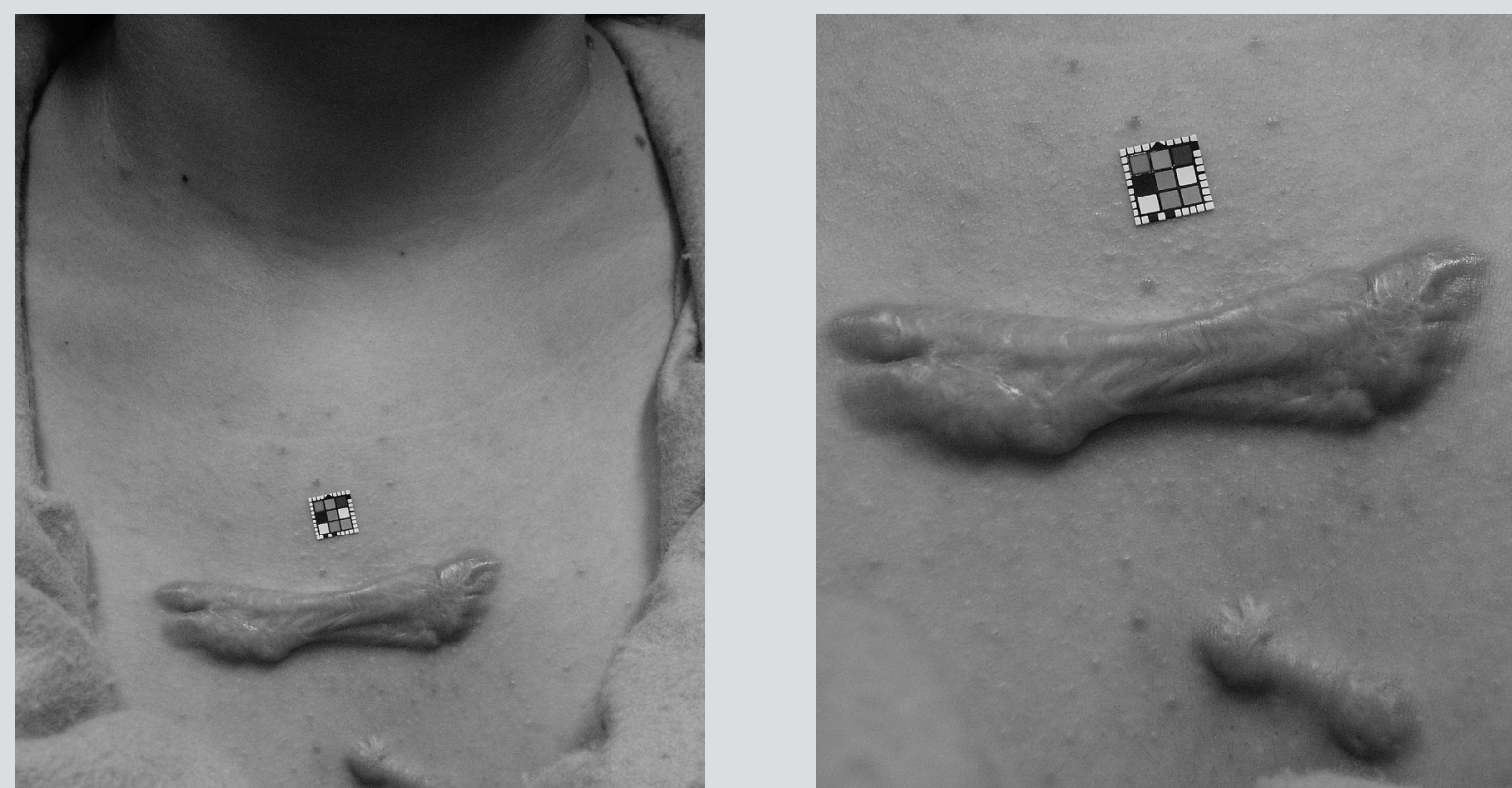

Fig. 5a: Case 4. Keloids of the chest, at initial presentation

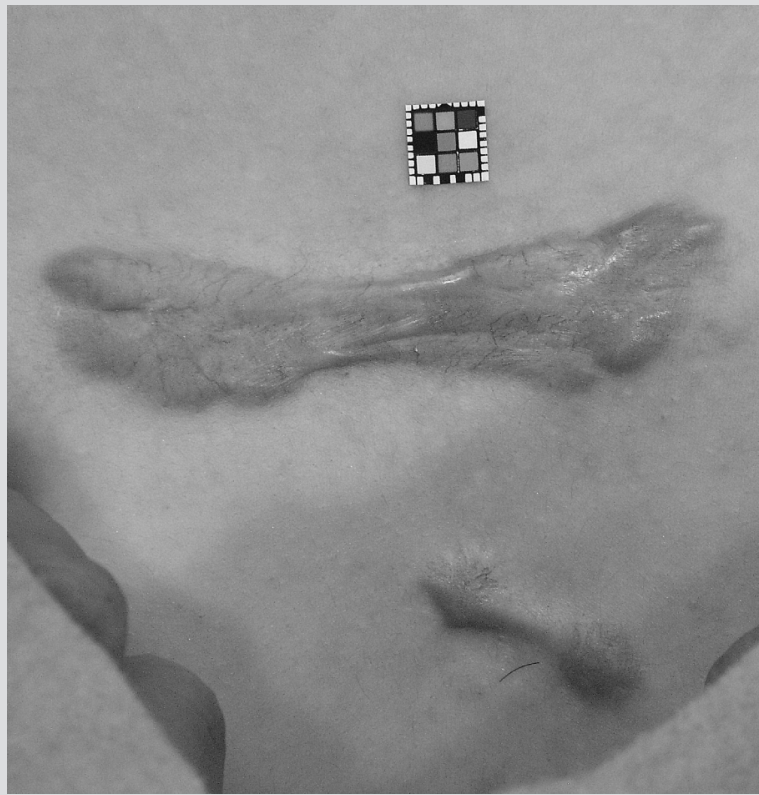

Fig. 5b: Case 4 . Keloids after $10^{\text {th }}$ treatment sessions 
seen and release of the contracture of the surrounding tissue allowed the surgeon who had referred her to us to surgically revise the scar after the $23^{\text {rd }}$ treatment session. Gradual release of the contracture of the lesion of the upper cheek was seen, as can be assessed by the release of the ectropion in fig. $\mathbf{4} \mathbf{b}$.

\section{Case 4. 31 year old female (fig.5a,b)}

Chief complaint: Keloid of the chest

Onset and course: This patient remembers having acne on the chest which turned into a keloid, gradually proliferating into the normal surrounding tissue during her mid 20's. She had previously been treated on numerous occasions at different clinics including local injections of corticosteroid. Although such treatments were sometimes transiently effective, immediate relapse and continuing proliferation of the lesion persisted. At presentation at our clinic she complained of strong pain on contact and itching of the lesion. The patient stated that there have been no dimensional changes for the year previous to presenting.

Initial findings: The keloid was located horizontally at the upper chest, centered on the skin overlying the sternum and measuring $10 \mathrm{~cm} \times 2 \mathrm{~cm}$. Elevation was highest at the lateral margin, measuring $1 \mathrm{~cm}$. This lesion was extremely hard and non-pliable.

Treatment: The patient was treated once every other week for 3 minutes per session. Topical steroids were prescribed. Although more repetitive treatments were advised, her schedule did not allow it. The treatment sessions were very painful for this patient. As seen in other severe keloid cases, even the softest pinch of the lesion caused pain and the firmness of the pinch had to be considerably lighter compared to other keloid patients. However, by the 10th treatment the decrease in elevation and rigidity of the lesion could be visualized. The patient is continuing treatment at this same interval.

\section{Discussion}

Low reactive level laser therapy was first described by Ohshiro in 1984. (1) Originally LLLT was used for pain attenuation in cases with general orthopedic arthrosis (2). It soon evolved to the application for the enhancement of wound healing (3) and as an anti-inflammatory agent. Further understanding and experience with LLLT has led to new treatments including LLLT for facial palsy, female infertility (4) and recently nerve regeneration (5). Treatment of hypertrophic scars and keloids with LLLT is not new to the authors. We have been using LLLT routinely since the mid 1980's and have been mentioning it throughout our reports on numerous occasions. Others have also been doing the same and it came as a complete surprise that there was not a single literary report on the subject in MedLine. The present study may be redundant to those who already use LLLT for the treatment of hypertrophic scars and keloids, but the authors hope that it may enlighten those who were unaware of this fact.

In the present study, we presented cases of LLLT in combination with topical corticosteroids or LLLT on its own as in Case 1. No cases in this report have been treated with local injection of corticosteroids, probably the strongest and most effective of conventional treatment methods, even though we routinely do so at our clinic. We have selected the cases to present the efficacy of LLLT as purely as possible, in order to prevent arguments that it was the corticosteroids that were the main therapeutic agent. All patients presented in this report had not received corticosteroid injections because they responded well to LLLT. One patient, Case 3, was able to undergo surgical revision of a hypertrophic scar once LLLT had softened the lesion and released contracture of the surrounding tissue after 23 treatment sessions. This patient was originally referred to our clinic by a plastic surgeon who deemed the lesion as inoperable at the time. This is a somewhat novel combination of LLLT and conventional treatment methods and requires further investigation and consideration in future patients.

The question remains as to whether all patients respond to LLLT in the same manner, and the answer is 'no'. There are patients who respond poorly, or respond to only a certain extent to LLLT for reasons unknown. There are cases where complete regression of the lesion is not possible and the effect of LLLT seems to stop at a certain level and injection of corticosteroids becomes necessary. In many cases, the lack of patient compliance may cause an imperfect or incomplete treatment effect. Many patients become reluctant to return to the clinic once their initial concerns such as pruritis or spontaneous pain have diminished. Often, these patients will return to the clinic once the symptoms return and will continue this cycle of treatment. This tendency is seen more in patients with keloids than those with hypertrophic scars. This is simply due to the difference in the nature of the lesions where keloids recur or become aggravated much more readily than hypertrophic scars.

The appealing features of LLLT for hypertrophic scars and keloids are the simplicity of the treatment itself and the lack of any associated risks and compli- 
cations of surgery or HLLT. A single treatment session takes only a few minutes and up to 10 minutes for patients with multiple lesions. However one facet of LLLT which may cause difficulty in patient compliance is the multiplicity of treatment sessions required for satisfactory results. This may become a burden, especially for busy patients, and may cause them to either drop out from treatment or continue treatment at suboptimal levels

On initial consultation we tell the patients that over 20 sessions will be required, which means that the patient will have to commute to the clinic for a period of over 5 months if treated once a week and over 10 months if treated once every other week. Concurrent use of topical steroids or local injections of steroids are advocated for most cases to expedite the regression of the lesions but in certain cases as in Case 1 in the present study, only LLLT is used when there is atrophic skin surrounding the keloid due to prolonged steroid use prior to presentation at our clinic. Treatment with LLLT can be somewhat painful for the keloid or hypertrophic scar patient. In this case the laser irradiation itself is completely sensation-free, but the firm pinching of the lesion during laser irradiation is painful. Pain is stronger in patients with harder, more rigid lesions than the softer more pliable lesions, and is strongest in patients who suffer from spontaneous pain of the lesion, and at their initial treatment. Pain subsides as treatment sessions continue as the lesions become softer and more pliable. Patients will notice changes in their lesions as early as after the second treatment concerning symptoms such as itch and pain, however change in cosmesis will take much longer.

Treatment goals differ for each patient. For those who suffer from itch and pain, alleviation of such symptoms may be enough, while for those seeking better cosmetic appearance, flattening of the lesions and resolution of dyschromia are required which is therefore more difficult and time-consuming to achieve.

Although the effect of low level laser irradiation has been elucidated at a cellular level by the works of Karu et al (6), and Kendrick-Smith (7), the systemic effect of LLLT and its physiology remain unclear. Since the pathophysiology of hypertrophic scars and keloids is not completely understood, how and why laser light exercises its therapeutic effect is even more perplexing. The authors hypothesize that hypertrophic scars and keloids are in an continual inflammatory condition of a wound, remaining in the acute phase of wound healing for some reason, resulting in an over-reaction in wound healing. The analogy holds true to the classic definition of inflammation as rubor, tumor, calor and dolor, i.e., redness, swelling, heat and pain. Young hypertrophic scars and all keloids are red, swollen and warm compared to normal skin and show symptoms such as itch and pain. Recent studies have reported that levels of transforming growth factor beta-1 ( TGF $\beta 1$ ) are increased in hypertrophic scars and keloids (8) and there is a model where the pathway of increased tumor necrosis factor alpha (TNF $\alpha$ ), a strong indicator of, if not responsible for, inflammatory responses, causes increased levels of TGFb1 (9). We believe that low incident levels of laser energy probably exert their effect through the modulation of such pathways for the alleviation of inflammation of acute trauma of the skin and the subsequent hypertrophic scars and keloids. Firm pinching of the lesion during laser irradiation to the lesion may create hypoxia and anemia of the lesion similar to that of silicon adhesive dressings (10).

Currently we have no physical evidence to uphold our hypothesis nor do we know whether the effect of laser is only local where local administration of the laser is required or whether laser irradiation to the neck as in the proximal priority treatment for pain (11) will show a favorable effect and exert the same response. We have recently started a study comparing local and proximal irradiation of the laser. As seen in Case 2 , so far, we have seen that laser irradiation of the neck while pinching the lesion shows similar therapeutic response however further evaluation is required to see whether there are any differences between the two methods of delivering laser energy to the body.

The sole aim of this report is to re-introduce LLLT for the treatment of hypertrophic scars and keloids. Prospective studies on local or proximal laser irradiation, retrospective analysis of past treatments are ongoing.

\section{Conclusion}

LLLT for the treatment of hypertrophic scars and keloids is a safe and effective method even for lesions resistant to conventional treatments of topical steroid and mechanical compression. The mechanism of laser treatment remains unclear but the authors believe that the anti-inflammatory effect of LLLT is the most probable cause of the regression of the lesions. Further basic studies are warranted for both LLLT and the pathophysiology of hypertrophic scars and keloids. 


\section{References}

1: Ohshiro T, Kato Y, Maeda $\mathrm{T}$ et al (1984): Application of the GaAlAs diode laser in the pain clinic. Journal of Japan Society of Laser Medicine and Surgery, 4(1): 191-192.

2: Shiroto C, Ono K, Ohshiro T (1989): Retrospective study of diode laser therapy for pain attenuation in 3635 patients: detailed analysis by questionnaire. Laser Therapy, 1(1):41-47.

3: Hawkins D, Abrahamse H (2005): Biological effects of helium-neon laser irradiation on normal and wounded human skin fibroblast. Photomedicine and laser surgery, 23(3):251-259.

4: Ohshiro T, Fujii S, Sasaki K et al (2001): Laser therapy as an adjunctive treatment for severe female infertility. A preliminary report. Laser Therapy, 11(2):96-102.

5: Rochkind S, Drory V, Alon M et al (2007): Laser phototherapy $(780 \mathrm{~nm})$, a new modality in treatment of long-term incomplete peripheral nerve injury: a randomized double-blind placebo- controlled study. Photomed Laser Surg, 25(5): 436-442

6: Ohshiro T, Maeda T (1992): Application of $830 \mathrm{~nm}$ diode laser LLLT as successful adjunctive therapy of hypertrophic scars and keloids. Laser Therapy, 4 (4): 155-168.

7: Karu, TI (1985): Biological action of intense visible monochromatic light and some of its medical applications. Laser (ed. by Galletti, G) :381.

8: Smiths, KC (1991): The photobiological basis of low level laser therapy. Laser Therapy, 3:19.

9: Camaner AB, Ferreira LM, Gragani A et al (2006): Upregulation of TGF-beta 1 expression may be necessary but is not sufficient for excessive scarring. J Invest Dermatol, 126(5): 1168-76.

10: Ohshiro T (2005): The proximal priority technique: How to maximize the efficacy of laser therapy, Laser Therapy; 14(3): 121-128 